\title{
Effect of Carbon Nanofiber Z-Threads on Mode-I Delamination Toughness of Carbon Fiber Reinforced Plastic Laminates
}

\author{
Kuang-Ting Hsiao ${ }^{1 *}$, Alexander M. Scruggs ${ }^{1}$, John S. Brewer Jr. ${ }^{1}$, Gregory J.S. Hickman ${ }^{1}$, Erin E. \\ McDonald $^{1}$, Kendrick Henderson ${ }^{2}$
}

\begin{abstract}
Delamination is a major drawback of carbon fiber reinforced plastics (CFRPs). Studies have reported that carbon nanofibers (CNFs) can improve the delamination toughness of various FRPs. However, lack of CNF alignment control caused substantial uncertainty in the improvements. In this study, a novel CNFs z-threaded CFRP (ZT-CFRP), which utilized z-aligned CNFs as long-range reinforcement threading through the packed carbon fiber bed, was manufactured. The mode-I delamination toughness $\left(\mathrm{G}_{\mathrm{IC}}\right)$ of the ZT-CFRPs was tested against both control CFRPs and unaligned CNF-modified CFRPs (UA-CFRPs). Through statistical comparison against control CFRPs, UA-CFRPs exhibited a relative change in mean $\mathrm{G}_{\mathrm{IC}}$ and coefficient of variation of $+13.99 \%$ and $+116.35 \%$, respectively, whereas the ZT-CFRPs of equivalent CNF concentration exhibited a relative change in mean $\mathrm{G}_{\mathrm{IC}}$ and coefficient of variation of $+28.93 \%$ and $-12.33 \%$, respectively. Accordingly, the CNF z-threads were found to play a positive role in toughening CFRPs, as supported by delamination experiments and microscopy analysis.

Keywords: A. Carbon fibres; A. Carbon nanotubes and nanofibres; B. Delamination; B. Fracture toughness
\end{abstract}

(C) 2016. This manuscript version is made available under the Elsevier user license http://www.elsevier.com/open-access/userlicense/1.0/ 


\section{Introduction}

\subsection{Background and Previous Works}

4 Given recent advances in composite manufacturing techniques, carbon fiber reinforced plastics (CFRPs)

5 have become commonplace in a wide variety of engineering applications due to their high modulus-to-weight

6 and strength-to-weight ratios. These traits make CFRPs particularly desirable to industries in which weight-

7 savings is paramount, such as the aerospace and automotive industries. However, unlike traditional metals,

8 CFRPs are anisotropic (i.e., the material properties are directionally-dependent). While CFRPs exhibit

9 desirable material properties in the direction of carbon fiber reinforcement, they are severely limited in the 10 through-thickness direction (i.e., z-direction), which lacks a reinforcing fiber network. Furthermore, CFRPs'

11 z-directional mechanical strength is substantially weakened by the carbon fiber-resin matrix interface and the 12 distinctly different mechanical properties between the rigid carbon fiber and relatively softer resin matrix. As

13 CFRPs are typically used as laminated structures, the absence of effective z-directional reinforcement results

14 in an inability to resist delamination when subjected to large out-of-plane mechanical loads.

15 Various methods have been studied to counteract delamination issues, however, the most common

16 methods involve either fiber stitching [1-3] or nanoparticle reinforcements [4-13], which can be subdivided

17 into interleaving and direct matrix dispersion approaches. Fiber stitching is costly and difficult for complex

18 and large CFRP part manufacturing, thus its applications are constrained by part-size, complexity, and budget.

19 Moreover, the stitching needles can break and interrupt the carbon fiber reinforcement, thereby reducing the

20 CFRP's compressive strength in the $x-y$ plane, particularly under hygrothermal conditions [3]. Therefore, the

21 improvements in interlaminar fracture toughness provided by fiber stitching also cause the reduction in other

22 critical material properties. Recently, nanoparticle reinforcements have also been used to provide significant

23 increases in interlaminar fracture toughness. However, many of the reported results suffered from substantial

24 increases in uncertainty $[4,5,6,11,12]$.

25 An increasingly prevalent research approach in the field of nanoparticle reinforcement is the insertion of 26 nano-reinforced resin layers (i.e., nanoparticle enhanced interlayers or interleaves) along the mid-plane of a

27 FRP laminate, which enhances interlaminar strengths [4-7]. Lee et al. [4] achieved mode-I delamination 28 toughness $\left(\mathrm{G}_{\mathrm{IC}}\right)$ increases of over $30 \%$ using a CNT-laden nanowoven carbon tissue (NWCT) interlayer. 
However, the coefficient of variation (i.e., CV, which is standard deviation divided by the mean) of the CNT-

2 NWCT was nearly nine-fold larger than the control CFRP. Arai et al. [5] increased $\mathrm{G}_{\mathrm{IC}}$ by approximately $50 \%$

3 using a CNF interlayer, yet their $\mathrm{G}_{\mathrm{IC}}$ plots illustrate that the samples with the CNF interlayer exhibited

4 significantly larger standard deviations than the control sample. Garcia et al. [6] grew a dense z-aligned CNT

5 forest on a silicon substrate, transferred the CNT forest to a prepreg, and then joined two carbon fiber prepreg

6 layers to form a through-thickness aligned CNT forest interlayer (i.e., interleaf); this method provided $\mathrm{G}_{\mathrm{IC}}$

7 improvement of approximately $50 \%$ and $150 \%$ for AS4/8552 and IM7/977-3 commercial prepregs,

8 respectively, along the CNT forest interlayer as compared to the baseline CFRP laminates. Although CNTs

9 and CNFs in nanoparticle-reinforced interlayers have yielded positive results, the increase in thickness,

10 weight, and complexity makes interleaf implementation between every interlaminar plane of a FRP laminate

11 less practical for lightweight composite designs and manufacturing consistency. However, it should be noted

12 that selective interleaving, as elaborated in $[6,7]$, may still provide a potential method of circumventing these

13 inherent shortcomings by strategically reinforcing critical locations rather than the entire structure.

14 Another common nanoparticle reinforcement approach is to uniformly disperse nanoparticles in the

15 matrix material throughout the FRP. This direct matrix dispersion approach is fundamentally different from

16 the interleaving approach because its purpose is to homogeneously improve the matrix's mechanical

17 properties and also enhance the FRP's interlaminar toughness, which is typically considered a matrix-

18 sensitive property. However, many challenges exist in the dispersion process and the processes used to

19 incorporate the nano-resin into the FRP system. Despite the challenges, CNTs and CNFs have been reported

20 to enhance the interlaminar strength of glass fiber reinforced plastics (GFRPs) via uniform dispersion in the

21 matrix material without adding any interleaves between GFRP laminae. A generic approach is to infuse the

22 nano-resin into a preform of dry fibers via Liquid Composite Molding (LCM) processes. Sadeghian et al. [8]

23 successfully infused $1 \mathrm{wt} \% \mathrm{CNF}$-modified polyester into GFRP laminates to improve interlaminar fracture

24 toughness by approximately $100 \%$. In their study, a CNF/polyester matrix was infused into the preform of

25 random glass fiber mats using Vacuum Assisted Resin Transfer Molding (VARTM). This study also

26 elaborated on nanoparticle filtration issues, which occurred as the CNF/polyester mixture flowed through the

27 glass fiber preform. As the CNF length (approximately 50-500 $\mu \mathrm{m}$ ) is much larger than the pore size within 
1 the glass fiber bed (glass fiber diameter $\sim 20 \mu \mathrm{m}$, so the pore size is of the same order of magnitude), certain

2 unaligned CNFs could be filtered by the glass fiber bed and gradually clog the flow channel, resulting in

3 uneven CNF concentration and a visible color gradient in the GFRP. Fan et al. [9] infused MWCNT-modified

4 epoxy into a preform of woven glass fiber mats via two different types of VARTM processes. The best

5 improvement in the interlaminar shear strength (ILSS) was $33 \%$, which was found in the $2 \mathrm{wt} \%$ MWCNT-

6 modified GFRP sample manufactured with injection and double vacuum assisted resin transfer molding

7 (IDVARTM). The IDVARTM utilized a step-wisely controlled atmosphere to (i) reduce the atmosphere and

8 permit more nano-resin being injected into the flow distribution medium layer under the vacuum bag and

9 above the glass fiber preform and (ii) subsequently increase the atmosphere to compact the nano-resin filled

10 flow distribution medium layer thus squeeze the nano-resin into the glass fiber preform that is under strong

11 vacuum. Although IDVARTM can handle higher MWCNT concentration than traditional VARTM,

12 IDVARTM was not able to create the preferable MWCNT alignment that traditional VARTM produced and

13 IDVARTM provided a lesser increase in ILSS at $0.5 \mathrm{wt} \%$ MWCNT concentration as well. The micrographs

14 provided within their study indicated that the short CNTs (compared with the gap between two adjacent glass

15 fibers in the pictures) had slightly preferred alignment along the flow direction while under a traditional

16 VARTM process but not under IDVARTM. Accordingly, Fan et al. concluded that the slightly preferred

17 MWCNT alignment contributed to the ILSS improvement in addition to MWCNT concentration.

18 Together, the accumulated literature suggests that lack of control over CNT/CNF alignment and filtration

19 could induce inconsistent composite quality. Furthermore, since carbon fibers (diameter $\sim 5 \mu \mathrm{m}$ ) are much

20 smaller than glass fibers (diameter $\sim 20 \mu \mathrm{m}$ ), the narrower gap between adjacent carbon fibers creates severe

21 challenges in terms of CNF or CNT alignment and filtration control; these issues are compounded for CNF

22 applications (compared with CNT applications), as CNFs are much longer (approximately 50-200 $\mu \mathrm{m}$ ) and

23 thicker (diameter $\sim 50-150 \mathrm{~nm}$ ) than CNTs (length $\sim 5-20 \mu \mathrm{m}$; diameter $\sim 1-20 \mathrm{~nm}$ ). As such, no significantly

24 successful studies regarding the infusion of CNF-modified resin in CFRPs have ever been reported.

25 To avoid the aforementioned challenges, electrophoretic deposition of water-dispersed short CNTs

26 (preferred length of 2-6 $\mu \mathrm{m}$, shortened from original length of $10-20 \mu \mathrm{m}$ by nitric acid treatment) on woven

27 carbon fabric, which was then dried and then stacked into a preform and infused with resin via VARTM 
1 process, has been studied [10] and produced an interlaminar shear strength increase of approximately $30 \%$.

2 However, handling the deposited CNTs on dry carbon fiber fabric could be cumbersome. Wicks et al. [11]

3 provided an alternative by growing CNTs in-situ on alumina fibers to create novel "fuzzy fiber reinforced

4 plastics" or FFRPs, which yielded a 76\% increase in mean $\mathrm{G}_{\mathrm{IC}}$ and an approximately two-fold increase in the

5 relative error (i.e., error/mean). Another FFRPs study by Wicks et al [12] illustrated the differences that CNT

6 length and epoxy grade (aerospace grade and marine grade) played in regards to $\mathrm{G}_{\mathrm{IC}}$. For the aerospace epoxy,

7 short CNTs actually reduced the $\mathrm{G}_{\mathrm{IC}}$ by $50 \%$, whereas the long CNTs increased the mean $\mathrm{G}_{\mathrm{IC}}$ by $32 \%$, while

8 also increasing the relative error by nearly $20 \%$ w.r.t. control. For the marine epoxy, short CNTs and long

9 CNTs increased the $\mathrm{G}_{\mathrm{IC}}$ by $89 \%$ and $100 \%$, respectively. However, short CNTs and long CNTs also increased

10 the relative error by $12 \%$ and $78 \%$, respectively. Due to the exposure to high temperatures and/or metal

11 catalysts, this process is not compatible with carbon fabrics. Moreover, the uncertainty in the improvements

12 may be a concern. As such, the FFRPs method still needs future improvements to make it more robust.

\subsection{Research Rationale}

14 This paper takes a notably different approach than the literature with respect to the nano-reinforcing 15 strategy, manufacturing process, and the attempt to gain an understanding of the roles of CNF alignment in 16 CFRPs. Based on insights from the aforementioned nano-resin VARTM literature [8], it is clear that CNFs 17 can potentially benefit z-directional strengths. However, multiple studies have suggested $[8,9]$ that poor CNF 18 alignment control and the aforementioned filtering effect could be responsible for reducing the effectiveness 19 of the CNF reinforcement or even, in some cases, weakening the composites. Although the works by Garcia 20 et al. [6] and Wicks et al. [11, 12] illustrated that both vertically-aligned CNTs in the interlayer and radially-

21 aligned CNTs deposited on individual alumina fibers can improve interlaminar fracture toughness, none of the 22 studies made a comparison against laminates with randomly distributed CNTs of the same ingredients. As 23 such, none of these previous works provided any direct proof that improving CNF alignment and filtering 24 issue could make the CFRP composite, of same ingredients, stronger. Nevertheless, it is reasonable to assume 25 that one could maximize CNFs' potential to reinforce the CFRP in the z-direction if one somehow aligns $26 \mathrm{CNFs}$ in the z-direction and reduces the chance of CNFs being filtered by the carbon fiber preform. 
Another interesting fact not yet discussed nor utilized explicitly in previous works is the unique scale relationship between the dimensions of CNFs with respect to the carbon fiber's diameter and the gap between

3 adjacent carbon fibers in a CFRP. Based on geometric argumentation, since the carbon fiber diameter is about

$4 \quad 5-7 \mu \mathrm{m}$, each z-aligned CNF (length $\sim 50-200 \mu \mathrm{m}$ ) is theoretically long enough to thread through an array of

5 carbon fibers and connect 7-100 carbon fibers. However, as carbon fibers are typically highly-packed in a

6 high fiber volume fraction CFRP, one must also account for possible filtration. A long CNF being flow-

7 transferred through the thickness of a highly packed carbon fiber bed will behave according to one or more of

8 three distinct scenarios: (A) the CNF will thread through the highly packed carbon fiber bed in the preferred

9 "zig-zag" or "wavy" path shown in Figure 1; (B) the CNF will entangle with other CNFs and become filtered

10 by the highly packed carbon fiber bed; (C) the CNF will lose the preferred z-direction orientation and rotate in

11 any direction within the $\mathrm{x}-\mathrm{y}$ plane, which will lead to it being filtered by the highly packed carbon fiber bed.

12 Note that scenarios B and C both increase the probability of additional CNFs being filtered during the flow-

13 transfer process, as they clog open channels in the packed carbon fiber bed. Two studies $[8,13]$ contributed to

14 additional understanding of the alignment and filtration process that occurs when a CNF or CNT is flow-

15 transferred through a fiber bed. First, from the VARTM filtering experiments of infusing CNF/polyester

16 mixture through random glass fiber mat preforms by Sadeghian et al [8], one can conclude that a thinner glass

17 fiber preform will filter fewer CNFs due to reduced compounded probability that CNFs will aggregate or

18 orientate within the x-y plane. Secondly, the microscopy flow experiments conducted by Fan and Advani [13]

19 illustrated that resin flow around a single glass fiber tends to align MWCNTs along the glass fiber's

20 circumference (similar to the streamline around a cylinder) and the resin flowing through a $100 \mathrm{~nm}$-wide

21 necking channel between two glass fibers tends to align MWCNTs in the direction of the channel.

22 Additionally, they suggested that shear flow dominated the MWCNTs' orientation instead of Brownian

23 motion. Furthermore, the study indicated that MWCNTs stayed in place once the resin stopped flowing.

24 By comparing the results from previous studies and trying to find a solution to address the

25 aforementioned issues of using CNFs in CFRPs, Hsiao and Hickman [14] proposed a new multiscale FRP

26 prepreg manufacturing method to incorporate z-aligned long carbon nanofibers dispersed in a B-stage resin

27 into a thin sheet of dry carbon fiber fabric by utilizing an electric field-induced CNFs alignment control in the 
1 B-stage resin followed by a non-isothermal unidirectional phase-changing CNF-resin flow transfer process.

2 This paper will discuss the manufacturing of the interesting CNFs z-threaded CFRP (ZT-CFRP) samples by

3 using the new multiscale FRP prepreg method [14] and the Out-of-Autoclave Vacuum-Bag-Only (OOA-

$4 \quad$ VBO) curing process. It will also investigate the statistical effectiveness of using CNF z-threads (or z-aligned

$5 \mathrm{CNFs})$ in a CFRP laminate to improve the mode-I delamination toughness $\left(\mathrm{G}_{\mathrm{IC}}\right)$. Due to the relatively long

6 and moderately dense CNF z-threaded reinforcement, if the CNF z-threads are effective and have the zig-zag

7 threading pattern as proposed in Figure 1, it is hypothesized that one could obtain significant and highly

8 repeatable improvements in $\mathrm{G}_{\mathrm{IC}}$ using a low concentration of CNFs. Additionally, as the CNFs are z-threading

9 through carbon fiber arrays, it is hypothesized that there would not be resin-rich interleaving regions in the

10 CFRP laminate. Therefore, any change in $\mathrm{G}_{\mathrm{IC}}$ would not be due to interleaving effect (resin-rich zone to blunt

11 the crack tip). Furthermore, it was hypothesized that unaligned long CNFs would reduce the delamination

12 toughness or make it unreliable as some unaligned CNFs could act as defects (described in aforementioned

13 scenarios B\& C) that encourage crack propagation. Note that this hypothesis will help to validate the widely-

14 accepted but not-yet-proven theory that unaligned CNFs or CNTs were responsible for inconsistent

15 reinforcement in many of the aforementioned publications. This study presents the mode-I delamination

16 experiments and the accompanying statistical analysis for CFRP samples constructed with CNF z-threads

17 (ZT-CFRPs), unaligned CNFs (UA-CFRPs), and unmodified epoxy resin (control CFRPs).

\section{2. Methods}

192.1 Materials

20 In this study, three 16-ply CFRP laminates were constructed for each of the four following sample types:

21 unmodified control CFRPs, CFRPs with $0.3 \mathrm{wt} \%$ (measured as a percentage of matrix weight) unaligned

22 CNFs (0.3wt\% UA-CFRPs), CFRPs with $0.3 \mathrm{wt} \%$ CNF z-threads ( $0.3 \mathrm{wt} \%$ ZT-CFRPs), and CFRPs with

$230.6 \mathrm{wt} \% \mathrm{CNF}$ z-threads ( $0.6 \mathrm{wt} \%$ ZT-CFRPs). The carbon fiber system used in the CFRPs was a Torayca T300

24 plain-weave fabric (203 g/ $\mathrm{m}^{2}$ areal weight, $3 \mathrm{~K}$ tow) acquired from Composite Fabrics of America. The

25 polymer matrix was a two-part epoxy from Momentive that consisted of Epon 862 resin (a diglycidyl ether of

26 bisphenol F) and an Epikure W catalyst (a non-MDA, aromatic amine curing agent containing

27 diethylmethylbenzenediamine). The CNFs were PR-24-XT-PS nanofibers from Pyrograf Products, Inc., 
1 which possess an average diameter of $100 \mathrm{~nm}$ and lengths ranging from $50 \mu \mathrm{m}$ to $200 \mu \mathrm{m}$ [15]. To increase experimental efficiency, only the inner four plies of the UA-CFRPs and ZT-CFRPs contained CNFs; this is

3 allowable since the delamination stress is concentrated on the centerline of the sample. A $60 \mu \mathrm{m}$-thick

4 polytetrafluoroethylene (PTFE) release film was inserted in the mid-plane of each CFRP to generate the 5 starter crack [16].

$6 \quad 2.2$ Control Prepreg Manufacturing

7 For each required stack of control prepreg (6-ply stacks for UA-CFRPs and ZT-CFRPs, 8-ply stacks for 8 unmodified CFRPs), a resin batch was produced by combining Epon 862 and Epikure W with a

9 stoichiometric ratio of 100:26.5, mechanically mixing the solution for five minutes, and then degassing the 10 mixture in a vacuum chamber a $40^{\circ} \mathrm{C}$ for one hour. A hand wet lay-up process was implemented to add the 11 degassed resin to individual $254 \mathrm{~mm} \times 254 \mathrm{~mm}$ sheets of woven carbon fabric. This process was repeated until 12 the prepregs had been stacked to the appropriate number of plies and the prepreg stack was placed on a hot 13 plate that had been preheated to $120^{\circ} \mathrm{C}$. After one hour of heating, the matrix reached the desired degree of 14 cure or "B-stage", and the prepregs were removed from the hot plate and transferred to a $-18^{\circ} \mathrm{C}$ freezer; the 15 prepregs were sealed inside a layer of nylon bagging to prevent moisture absorption during storage. The 16 desired B-stage allows the resin to remain solid at room temperature and flow at approximately $50^{\circ} \mathrm{C}$; the 17 amount of heating time required to achieve this B-stage was determined through trial-and-error.

\section{$18 \quad 2.3$ UA-CFRP Prepreg Manufacturing}

19 To form the uniformly dispersed CNF/epoxy nano-resin system (i.e., CNF/epoxy mixture), first, the 20 CNFs were weighed and added to the appropriate amount of Epon 862, then combined using a magnetic 21 stirrer. The mixture then underwent one hour of high-shear mixing by a Stir-Pak Laboratory Mixer operating 22 at $900 \mathrm{rpm}$, followed by one hour of agitation in a QSONICA Q700 sonicator. A small sample taken from the 23 resin was inspected under a microscope to ensure the aggregations were broken apart. Having disentangled

24 the majority of CNF agglomerations, the designated amount of Epikure $\mathrm{W}$ was added to the solution,

25 magnetically stirred for five minutes, and then degassed in a vacuum chamber for one hour at $80^{\circ} \mathrm{C}$. The UA-

26 CFRP prepregs followed the same hand wet lay-up process as the control prepregs, however, the B-stage UA-

27 CFRP prepregs were packaged and stored at $-18^{\circ} \mathrm{C}$ as individual plies instead of stack of plies. 


\subsection{Novel ZT-CFRP Prepreg Manufacturing}

The CNF/epoxy mixture for the ZT-CFRP prepregs was first prepared using the same procedure as that used for the UA-CFRP prepregs. However, to obtain the novel ZT-CFRP prepreg, several additional steps are needed according to [14] and the research rationale discussed in Section 1.2. Figure 2 summarizes the process into four major steps as explained hereafter. (1) Introduce well-dispersed CNF/epoxy mixture into a thin sponge sheet to bear the nano-resin; note that the CNFs are randomly orientated at this step. (2) Use an electric field to align CNFs inside the thin sponge in the thickness direction and then rapidly cool the B-stage epoxy into solid-phase to lock the z-alignment of CNFs. (3) Apply a non-isothermal, phase-changing, unidirectional resin-transfer process tor thread the z-aligned CNFs along with the resin flow into a preheated thin carbon fabric sheet. Note that during the non-isothermal phase-changing flow process, it is imperative to cool down and keep the majority of B-stage epoxy within the sponge solidified to lock the preferred CNF zalignment. As such, the epoxy only starts to melt upon contact with the preheated carbon fabric, allowing all CNFs to start from a very well controlled z-aligned angle to thread through the carbon fiber array of the carbon fabric. After the pre-z-aligned CNFs enter the carbon fabric with a good entrance angle, the flowinduced alignment phenomena described in [13] will keep the CNFs flowing along the tangent of the circumference of each carbon fiber and the direction of flow channel formed by the gap of adjacent carbon fibers. In other words, this shear flow-induced alignment inside the packed carbon fiber bed will help the prez-aligned CNFs thread through the carbon fiber array inside the carbon fabric. To reduce the probability and negative impact of undesired CNF filtering effect caused by imperfectly-aligned CNFs, imperfectly dispersed CNFs (such as CNF aggregates), or blockages in the flow channels (i.e. scenarios B \& C described in section 1.2), a thin carbon fabric sheet is preferred to a thick preform; thus, a thin prepreg is appropriate considering the nature of all involved physics. (4) After completing the CNF z-threading process, the carbon fabric sheet is cooled down to solidify the B-stage epoxy and obtain the ZT-CFRP prepreg.

The detailed process to implement the four major steps (illustrated in Figure 2) on the specific epoxy resin, CNFs, and carbon fabric system used in the mode-I delamination experiments are given as follows:

Step 1: After degassing, the prepared CNF/epoxy mixture was added via hand wet lay-up to a $254 \mathrm{~mm} \mathrm{x}$ $254 \mathrm{~mm}$ sheet of nonwoven polyester breather material (henceforth referred to as the "sponge") that had been 
1 placed inside the confines of a parallel plate capacitor; prior to the lay-up process, the capacitor was preheated

2 to $120^{\circ} \mathrm{C}$ on a hot plate and the top and bottom surfaces of the sponge were covered with a PTFE film.

3 Step 2: The resin-saturated sponge was then heated for 39 minutes before it was subjected to a 0.67

$4 \mathrm{kV} / \mathrm{mm}$ electric field at $6 \mathrm{~Hz}$ for 60 seconds, which aligned the CNFs in the z-direction of the sponge sheet;

5 the waveform was a DC pulse with a 50 percent duty cycle. The z-alignment was achieved because the

6 electric field polarized the CNFs. This polarization created dipole moments at the opposing ends of the CNFs,

7 which caused them to rotate and align lengthwise between the opposing plates of the capacitor. After

8 completing the electric field induced $\mathrm{CNF}$ alignment process, the sponge saturated with B-stage resin

9 containing z-aligned CNFs was rapidly cooled to $5^{\circ} \mathrm{C}$ to solidify the B-stage epoxy; the solidified B-stage

10 epoxy ensured the CNFs remained in the preferred z-aligned orientation.

11 Step 3: Once the sponge had been thoroughly cooled, it was removed from the capacitor, centered

12 between two layers of porous PTFE-coated fiberglass, and then placed atop a sheet of carbon fabric that was

13 placed on top of a porous PTFE-coated fiberglass, a layer of polypropylene flow distribution media, and a

14 supporting aluminum plate. A $254 \mathrm{~mm} \times 254 \mathrm{~mm}$ caul plate was then placed on top of the sponge, the layers of

15 peel ply were trimmed to the edges of the caul plate, and then the edges of the caul plate were constrained

16 with a dam to ensure the unidirectional resin transfer occurred in the thickness direction. Two vacuum

17 channels equipped with vacuum lines were then placed on top of the flow distribution media and sealant tape

18 was placed on the edges of the aluminum mold to anchor a layer of vacuum bagging (see Fig. 2 for the setup).

19 After vacuum (approximately 1 bar) was drawn on the samples, the mold was placed in an opened hot press

20 that had the bottom plate preheated to $120^{\circ} \mathrm{C}$ (the top plate was not needed for this step so it was unheated and

21 did not apply any compaction pressure to the mold). After ten minutes, the resin completed the transfer from

22 the sponge into the carbon fabric and the resin had been heated long enough to achieve the same level of B-

23 stage as the control CFRP and UA-CFRP prepregs. During this step, the individual as well as the integrated

24 influences of (i) the one-sided heating and one-side cooling arrangement, (ii) the significant thermal

25 conductivity difference between the resin system and the carbon fiber fabric, and (iii) the B-stage resin's

26 ability to rapidly change between solid phase and liquid phase during the non-isothermal z-directional flow

27 transfer process, were paramount to the success of the CNF z-threading through the carbon fiber array (i.e., 
1 packed carbon fiber bed). The CNF/epoxy mixture (largely composed of epoxy) has a relatively low thermal

2 conductivity compared with compacted carbon fabric. This distinct thermal conductivity difference is helpful

3 to maintain the majority of epoxy inside the sponge in solid phase and all the epoxy inside the carbon fabric in

4 liquid phase, which maximizes the chance of z-threading CNFs through the carbon fabric.

5 Step 4: Upon completing the z-threading process, the ZT-CFRP prepreg was cooled down to room

6 temperature and individually packaged and stored at $-18^{\circ} \mathrm{C}$ according to the same procedure used for the UA-

7 CFRP prepregs.

$8 \quad 2.5$ Laminate Sample Preparation

9 The control CFRP samples were stacked for curing in the following order: an 8-ply control CFRP

10 prepreg, a PTFE film (used to form the precrack), and then another 8-ply control CFRP prepreg. The UA-

11 CFRP samples had the following stacking sequence: a 6-ply control CFRP prepreg, two single-ply UA-CFRP

12 prepregs, a PTFE film, another two single-ply UA-CFRP prepregs, and then a final 6-ply control CFRP

13 prepreg. The ZT-CFRP samples used the same general stacking sequence as the UA-CFRPs.

14 After stacking, the prepregs were placed on a release agent-coated aluminum plate and then the prepregs

15 were covered by a layer of peel ply and two layers of distribution media. Two vacuum channels equipped

16 with high-temperature vacuum lines were placed on top of the distribution media and then sealant tape was

17 placed on the edges of the aluminum mold to anchor a layer of vacuum bagging. After vacuum was drawn on

18 the samples, the mold was placed in a hot press and the heated plates were closed until only a small gap

19 remained between the top heating plate and the aluminum mold; this emulated an even-heating oven

20 environment. The OOA-VBO cure cycle consisting of vacuum application, temperature, and timing used for

21 curing these samples is shown in Table 1.

22 After demolding, the precrack tip location (i.e., the leading edge of the PTFE film) was marked on the

23 face of CFRP to ensure the samples were cut orthogonal to the precrack edge. The targeted dimensions for the

24 trimmed delamination testing samples were: $153 \mathrm{~mm}$ length, $50 \mathrm{~mm}$ initial precrack length, $25 \mathrm{~mm}$ width, and

25 approximately $3 \mathrm{~mm}$ thick. Once cut, the samples were polished, the edges of the samples were coated with

26 white acrylic paint, and then graduations were scribed in the paint using a fine-tipped razor blade. 
The mode-I interlaminar fracture toughness for each sample was evaluated using the double cantilever

3 beam (DCB) method in accordance with ASTM D-5528 [16]. ASTM D-5528 was originally intended for

4 testing unidirectional fiber systems; however, the standard also elaborates that woven fabrics can be used with

5 the understanding that they tend to experience run-arrest phenomena as a result of resin rich pockets between

6 the warps and wefts of the weave.

7 Prior to testing, the surfaces of two aluminum loading blocks and a small portion of the leading edge of

8 each sample surface were sanded and then the blocks were bonded to the sample. After the adhesive cured,

9 the samples were loaded into a MTS 810 universal testing machine equipped with a $501 \mathrm{~b}(\sim 222 \mathrm{~N})$ load cell;

10 the experimental testing setup is shown in Figure 3. Adhering to the standard testing procedure, the samples

11 were loaded at a crosshead speed of $1 \mathrm{~mm} / \mathrm{min}$ until the crack had progressed $3-5 \mathrm{~mm}$ from the precrack tip,

12 then they were unloaded and the precrack tip location was marked on both sides of the sample. The samples

13 were then reloaded at a crosshead speed of $1 \mathrm{~mm} / \mathrm{min}$ until the crack had progressed at least $50 \mathrm{~mm}$ from the

14 precrack tip. The crack location was tracked using a high-speed digital camera and the displacements were

15 noted via the graduations on the sample's edge. The displacement data was combined with the load cell

16 output to create load vs. displacement plots for each test.

17 The Modified Compliance Calibration (MCC) Method was used to interpret the interlaminar fracture

18 toughness, $\mathrm{G}_{\mathrm{IC}}$, from the load vs. displacement data; this is shown in Eq. 1.

19 In Eq. $1, P$ is the applied load, $C$ is the compliance $(\mathrm{C}=\delta / \mathrm{P}), \delta$ is the crosshead displacement, $b$ is the sample

20 width, $h$ is the sample thickness, and is an experimentally determined correction factor found by the least

21 squares linear fit of the delamination length normalized by sample thickness (i.e., $a / h$ ) as a function of the

22 cube root of compliance (i.e., $C^{l / 3}$ ), wherein is the slope of the linear plot of $a / h v s C^{1 / 3}[16]$.

232.7 Carbon Fiber Volume Fraction

24 To further explore the differences between the samples, the fiber volume fractions were determined by 
$v f=n * A w \rho f * t$

1 , where $n$ is the number of plies, $A w$ is fiber areal weight $\left(203 \mathrm{~g} / \mathrm{m}^{2}\right), \rho f$ is fiber density $\left(1.78 \mathrm{~g} / \mathrm{cm}^{3}\right)$, and $t$ is

2 laminate thickness measured for each sample. The carbon fiber volume fractions for the control CFRPs,

$30.3 \mathrm{wt} \%$ UA-CFRPs, $0.3 \mathrm{wt} \%$ ZT-CFRPs, and $0.6 \mathrm{wt} \%$ ZT-CFRPs were $53.41 \%, 52.14 \%, 52.65 \%$, and

$452.52 \%$, respectively. The carbon fiber volume fractions of all samples are very close. Nevertheless, the slight

5 differences could be due to either thickness measurement errors, manufacturing difference (such as B-stage

6 resin control and prepreg resin content control), or difference in flow resistance while the excess resin was

7 squeezed out from the CFRP prepreg during the OOA-VBO curing cycle. Theoretically, the presence of

8 CNFs, either z-threaded or not, should add resistance against the resin flow in the carbon fiber bed and the

9 compaction process during OOA-VBO curing. Further analysis regarding the compaction process, either

10 using OOA-VBO or autoclave curing, could be the aim of future investigations.

\subsection{Microscopic Characterization}

12 The overall laminate structures and delaminated surface morphologies of the CFRP samples were 13 characterized and compared using a Nikon Eclipse LV150 optical microscope (optical magnification range

14 100-3000x) equipped with an extended depth of focus (EDF) module. Additionally, a JEOL JSM-IT100

15 scanning electron microscope (SEM) was used to further explore the structure of the CNF z-threads network.

162.9 Laminate Structure Investigation

17 As described in previous sections, the CFRPs used in this study did not insert any interleaf film within 18 their structures. Furthermore, it was hypothesized (Section 1.2) that no resin-rich layers similar to an interleaf

19 were unintentionally formed during the CFRP manufacturing process; therefore, one will be able to directly

20 relate the $\mathrm{G}_{\mathrm{IC}}$ change to the role of CNFs instead of mixed roles between resin-rich interleaves and CNFs.

21 Note that an interleaf is typically a thick thermoplastic film (approximately $100 \mu \mathrm{m}$ thick) that absorbs

22 substantial plastic deformation at the crack tip during delamination, which blunts the crack tip to toughen a

23 FRP laminate [7]. To verify the hypothesis that no interleaving layers exist in any of the CFRPs tested in this

24 study, the as-produced layer-by-layer laminate structures were investigated using side-view optical

25 micrographs (Figure 4) acquired from the upper half of the symmetric CFRP laminates right above the mid-

26 plane region (annotated as region "2") where the precrack was formed (annotated as region " 3 "). As the 
1 laminates are composed of plain-weave carbon fabric, one can distinguish the interlaminar boundary between

2 individual plies within the laminate by finding the regions (i.e., region "1") where the warps from one ply

3 merges with the wefts of the subsequent ply. If these laminates had resin-rich interleaves, either inserted or

4 unintentionally generated during manufacturing, these warps and wefts in region " 1 " would never merge since

5 they would be separated by a resin-rich interleaf layer as discussed in [7]. Upon examination of Figure 4, one

6 can determine that there are no such resin-rich layers present between the plies of the CFRP laminates used in

7 this study. Therefore, any observed differences in $\mathrm{G}_{\mathrm{IC}}$ can be solely attributed to the role of CNFs in the tested

8 CFRPs.

\section{3. Results \& Discussion}

10 Figure 5 shows the load $(P)$ vs. displacement $(\delta)$ plots for each of the four sample types and Figure 6

11 shows the $\mathrm{G}_{\mathrm{IC}}$ (calculated by using Eq. 1) vs. delamination length (a). Table 2 presents a descriptive statistical

12 summary comparing the performance of each respective sample type. The $\mathrm{G}_{\mathrm{IC}}$ values in Table 2 were

13 obtained by averaging all $\mathrm{G}_{\mathrm{IC}}$ data, calculated by Eq. (1) for all $P \& \delta$ data sets with the curve-fitted $A_{l}$, after

14 peak load for each sample; the statistics for each laminate type were computed from the results. Further

15 discussion based on Figure 5, Figure 6, and Table 2 will be given in the following sections.

$16 \quad 3.1$ Control CFRP

17 As shown in Figures 5a and 6a, the load vs. displacement plots and $\mathrm{G}_{\mathrm{IC}}$ data for all the control CFRP

18 specimens were consistent between individual tests. It is important to note that one of the control samples was

19 manufactured with a longer initial crack length, which yielded a longer total delamination length than the

20 other samples. However, this variation was accounted for in the compliance and GIC calculations; this is

21 confirmed in Figure 6a, wherein the delamination length is longer, yet the GIC values are consistent with the

22 other samples. In subsequent samples, this initial crack length was shortened to the targeted dimension to

23 reduce the time required to run each test. In Figure 5a, the control CFRPs displayed the typical run-arrest

24 phenomena of woven fiber systems. Despite this erratic behavior, the $\mathrm{G}_{\mathrm{IC}}$ results in Table 2 reveal that the

25 mean and $\mathrm{CV}$ for the control samples were $305.33 \mathrm{~J} / \mathrm{m}^{2}$ and $4.15 \%$, respectively. Figure 7 a displays an optical

26 micrograph of the delamination surface of a control CFRP sample; the clean and smooth surface indicates that

27 the delamination propagated through the matrix without interruption, leaving the carbon fibers relatively 
1 undamaged. This is as expected for CFRP laminates constructed from plain-weave fabric, which, unlike

2 unidirectional CFRPs, do not possess fibers bridging inside the crack length.

$3 \quad 3.20 .3 w t \% U A-C F R P$

4 As shown in Figure 5b, the UA-CFRP specimens also experienced the run-arrest phenomena. Although

5 the results in Table 2 show an approximate $13.99 \%$ increase in mean $\mathrm{G}_{\mathrm{IC}}$ value $\left(348.06 \mathrm{~J} / \mathrm{m}^{2}\right.$ ), the unaligned

6 CNF-reinforcement also added a relative increase in the CV by $116.35 \%$ w.r.t the control. The observed

7 inconsistencies between individual sample tests occurred because of the lack of CNF alignment control, as

8 hypothesized in Section 1.2. As the alignment of the CNF reinforcement is uncontrolled in the UA-CFRPs, it

9 is possible that some CNFs provided local toughening to the laminate by pseudo-aligning in the through-

10 thickness direction. However, some other CNFs that did not experience this pseudo z-alignment created

11 nonhomogeneous defects between the carbon fibers in the form of CNF bundles (a non-uniform network of

12 unaligned $\mathrm{CNFs}$ ) or in-plane alignment. Unlike z-aligned CNFs, CNFs with in-plane alignment do not

13 contribute their fiber strength to the interlaminar fracture toughness; this reduces local bonding strength in the

14 z-direction, while providing unnecessary laminate reinforcement in the in-plane directions that are reinforced

15 by the continuous carbon fabric. Figure $7 \mathrm{~b}$ displays an optical micrograph of the delamination surface of one

16 of the $0.3 \mathrm{wt} \%$ UA-CFRPs. Like the control sample, the UA-CFRP delamination surface is also clean and

17 smooth. As previously suggested, most CNFs are not z-aligned nor pseudo-z-aligned, therefore they become

18 defects that encourage delamination; this phenomena explains the smooth fracture surface morphology. At the

19 delamination surface, a CNF z-thread (i.e., z-aligned CNF) in the CFRP should have one root in the matrix, it

20 should extrude from the root, and it should end with one broken tip as further explained in Figure 8. The local

21 angle of a CNF z-thread may not be perfectly perpendicular to the delamination surface due to the zig-zag

22 threading pattern through the highly packed fiber bed. Any CNF formation other than the "one-root and one-

23 broken-tip" is not a z-thread. If one uses this "one-root and one-broken-tip" rule to examine the UA-CFRP

24 delamination surface in Figure 7b, one can find that most of the observed CNFs are not z-threads since they

25 either have two roots or are submersed in the matrix parallel to the delamination surface. 
As expected, Figure $5 \mathrm{c}$ confirms that the ZT-CFRPs experience the run-arrest phenomena in the load vs.

3 displacement plots due to the plain-weave carbon fabric. However, in comparison to the control CFRPs and

4 the $0.3 \mathrm{wt} \%$ UA-CFRPs, the $0.3 \mathrm{wt} \%$ ZT-CFRPs experienced less severe run-arrest events. Additionally, both

5 Figures $5 \mathrm{c}$ (load vs. displacement) and $6 \mathrm{c}\left(\mathrm{G}_{\mathrm{IC}}\right.$ vs. crack length) illustrate more consistent results between all

$6 \quad 0.3 \mathrm{wt} \%$ ZT-CFRP specimens. According to the data presented in Table 2, the mean $\mathrm{G}_{\mathrm{IC}}$ value $\left(393.67 \mathrm{~J} / \mathrm{m}^{2}\right)$

7 increased by approximately $28.93 \%$ while reducing the CV by $12.33 \%$ w.r.t. the control. As such, in

8 comparison to the control CFRPs, the ZT-CFRPs are significantly stronger and more reliable.

9 Comparing the UA-CFRP and ZT-CFRP results, as the testing setup and the CNF concentration were the

10 same between the two types of CFRPs, the statistical improvements in the $\mathrm{G}_{\mathrm{IC}}$ in terms of mean value and CV

11 can be solely attributed to the role of controlled CNF alignment in the CFRP (i.e., CNF z-threads vs.

12 unaligned CNFs). Furthermore, the increased repeatability between all $0.3 \mathrm{wt} \%$ ZT-CFRP tests provides a

13 more predictable fracture pattern, which could lead to better predictive models that help reduce the risk of

14 catastrophic failures in products or systems that implement woven-fabric CFRPs.

15 Figure $7 \mathrm{c}$ displays an optical micrograph of the delamination surface of one of the $0.3 \mathrm{wt} \%$ ZT-CFRP

16 samples, which contains organized CNFs extending from beneath the carbon fiber tows. Additionally, other

17 CNFs have been pulled out and/or fractured. The epoxy matrix under the carbon fibers was severely fractured

18 due to the rooted CNF z-threads that extend from beneath the carbon fiber array. This observation illustrates

19 the reason why the ZT-CFRP samples exhibited higher $\mathrm{G}_{\mathrm{IC}}$ values: it takes additional energy to fracture and

20 pull-out deeply-embedded, long (originally, CNF length is 50-200 $\mu \mathrm{m}$ [15]) carbon nanofibers threading

21 through the array of carbon fibers and the matrix surrounding them. It is interesting to note that all of the

22 micrographic features observed in Figure $7 \mathrm{c}$ can also be explained from the proposed schematic of CNF z-

23 threading patterns shown in Figure 8 and Figure 1. In addition to optical microscopy, Figure 9 depicts a

24 similar delamination surface of a $0.3 \mathrm{wt} \%$ ZT-CFRP using scanning electron microscopy (SEM), which

25 provides a higher resolution view than Figure 7c. As shown in the SEM micrograph, many of the fractured

26 CNF z-threads protrude with very short length from the matrix material, which highlights two important

27 ideas. First, this allows us to realize that many fractured CNF z-threads with extremely short pull-out lengths 
1 are practically impossible to distinguish by optical microscopy alone, and, second, the short protruding length

2 (i.e., CNF pull-out length) signifies a strong and effective bond (maybe also due to the mechanical interlock

3 caused by the CNF's zig-zag threading pattern in the packed carbon fiber bed) between CNF and the epoxy in

4 the novel ZT-CFRP laminate. Very complex fracture surface near the roots of broken CNFs indicate the CNF

5 z-threads caused further complex fracture in the matrix when they were broken. Furthermore, the CNF z-

6 threading observed in the optical and SEM micrographs illustrates that the new prepreg manufacturing

7 process creates an organized network of CNF z-threads within the ZT-CFRPs; this observation explains the

8 repeatability of results. One can compare Figure 7c, Figure 8, and Figure 9 and find consistent geometrical

9 features of this novel ZT-CFRP laminate. By comparing the $\mathrm{G}_{\mathrm{IC}}$ results and the microscopy analysis between

10 the ZT-CFRPs and the UA-CFRPs with identical CNF concentration, one can conclude that controlled CNF

11 alignment within the CFRP is critical to the delamination fracture toughness.

$123.40 .6 w t \% Z T-C F R P$

13 As shown in Figure 6d, the $0.6 \mathrm{wt} \%$ ZT-CFRPs were relatively consistent between tests in terms of $\mathrm{G}_{\mathrm{IC}}$

14 value, yet Figure 5d displays rather erratic run-arrest cycles that are partially comparable to, but less severe

15 than, those seen in the $0.3 \mathrm{wt} \%$ UA-CFRPs. Despite doubling the CNF weight percentage, the mean $\mathrm{G}_{\mathrm{IC}}$ value

16 for the $0.6 \mathrm{wt} \%$ ZT-CFRPs $\left(371.54 \mathrm{~J} / \mathrm{m}^{2}\right)$ fell below that of the $0.3 \mathrm{wt} \%$ ZT-CFRPs $\left(393.67 \mathrm{~J} / \mathrm{m}^{2}\right)$. However,

17 relative to the control CFPRs, the $\mathrm{G}_{\mathrm{IC}}$ mean increased by $21.69 \%$ and the $\mathrm{CV}$ increased by $43.46 \%$.

18 The disparity between the results for the $0.3 \mathrm{wt} \%$ ZT-CFRPs and $0.6 \mathrm{wt} \%$ ZT-CFRPs comes from issues

19 with nanoreinforcement non-homogeneity. Although CNF aggregations are mostly broken apart with the

20 combined high-shear mixing and sonication regime, as CNF concentration increases, the van der Waals forces

21 between the nanofibers increase, creating a greater tendency for CNFs to aggregate. These aggregations

22 diminish the performance of the alignment process, which inevitably results in laminates with inconsistent

23 CNF alignment structures. Figure 7d highlights the extent of these CNF aggregations observed in the $0.6 \mathrm{wt} \%$

24 ZT-CFRPs. Figure 10 further depicts the role CNF concentration plays in the CNF alignment control within a

25 liquid epoxy throughout the electric field induced alignment process, which elaborates the reason the $0.3 \mathrm{wt} \%$

26 ZT-CFRP had a more consistent CNF alignment structure. The orientation distribution function (ODF)

27 method of nanoparticle alignment characterization described by Fan and Advani [13] was used to illustrate the 
1 orientation state of the CNFs within the liquid epoxy during the electric field application process by utilizing

2 the components of a second order orientation tensor in two dimensions to draw an ellipse [13] representing

3 the CNF alignment. The orientation state ellipses generated by this method provide an indication in terms of

4 both average CNF rotation angle and degree of alignment through the respective ellipse rotation and the size

5 relation between the major and minor axes of the ellipse. For the latter, two extreme cases exist: if the ellipse

6 is circular, the alignment is completely random, whereas, if the ellipse forms a line, the alignment is perfect.

7 As the alignment process reveals in Figures 10a, 10b, and 10c, (also, see video " $0 . \mathbf{3 w t} \%$ Alignment

8 Video 5X Speed.mp4") the $0.3 \mathrm{wt} \%$ sample achieved nearly perfect alignment in epoxy resin within 30

9 seconds of applying the electric field (i.e., the ellipse formed into a line that was rotated in the direction of the

10 applied electric field), whereas the $0.6 \mathrm{wt} \%$ sample (shown in Figure 10d, 10e, and $10 \mathrm{f}$ (also, see video

11 “0.6wt\% Alignment Video 5X Speed.mp4”) never surpassed a mediocre measure of alignment. The less

12 effective electric field induced CNF z-alignment step (i.e., step 2 in Figure 2) subsequently caused less

13 controlled z-threading process (i.e., step 3 in Figure 2) during the prepreg manufacturing, which explains the

14 lack of increase in $\mathrm{G}_{\mathrm{IC}}$ improvement at $0.6 \mathrm{wt} \% \mathrm{CNF}$ concentration. It is an aim of future works to improve the

15 electric field alignment process to make it capable of aligning higher CNF concentrations.

\section{4. Conclusions}

17 To evaluate the effect of CNF z-threads on the mode-I delamination toughness of CFRPs, four sample

18 types were constructed: control CFRPs, $0.3 \mathrm{wt} \%$ UA-CFRPs, $0.3 \mathrm{wt} \%$ ZT-CFRPs, and $0.6 \mathrm{wt} \%$ ZT-CFRPs.

19 The comparison among the control CFRPs, the $0.3 \mathrm{wt} \%$ UA-CFRPs, and the $0.3 \mathrm{wt} \%$ ZT-CFRPs proves that

20 controlled CNF alignment in CFRPs has a significantly positive contribution to the mode-I delamination

21 toughness $\mathrm{G}_{\mathrm{IC}}$ (the mean and the $\mathrm{CV}$ ) and further lessens the severity of the run-arrest events typically

22 associated with CFRPs made of plain-weave carbon fabrics. However, comparisons between the $0.3 \mathrm{wt} \% \mathrm{ZT}$ -

23 CFRPs and 0.6wt\% ZT-CFRPs revealed a decreasing trend in $\mathrm{G}_{\mathrm{IC}}$ improvement at the higher CNF

24 concentration. This phenomenon was caused by nonhomogeneous CNF dispersion at higher CNF

25 concentration, which led to CNF agglomerations that diminished the CNF alignment process. Further

26 microscopy analysis shows completely different fracture morphologies of the four types of CFRPs. A

27 comparison by integrating the $\mathrm{G}_{\mathrm{IC}}$ data and microscopy of the $0.3 \mathrm{wt} \%$ UA-CFRPs and $0.3 \mathrm{wt} \%$ ZT-CFRPs 
1 shows that CNF z-alignment can totally change the role of CNFs in a CFRP. For a high fiber volume fraction

2 CFRP, the z-aligned CNFs thread through the packed carbon fiber bed in a zig-zag (or wavy) pattern and

3 become z-threads in the CFRP, which helps create a complex and deep matrix fracture near the root of each

4 broken $\mathrm{CNF}$ z-thread during the mode-I delamination process; therefore, more energy is consumed during the

5 delamination. On the contrary, unaligned CNFs contribute to a smooth delamination surface and act as defects

6 that encourage delamination.

7 This study illustrated that CNF z-threads can significantly improve the delamination performance of

8 CFRPs. It also discussed the background issues to overcome, as well as the novel and detailed methodology

9 used to manufacture CNF z-threaded CFRP prepreg. It is hopeful that further research into CNF dispersion

10 and alignment improvement could produce ZT-CFRPs that utilize much higher CNF concentrations to

11 provide dramatically higher mean $\mathrm{G}_{\mathrm{IC}}$ values and very low coefficients of variation, which would be

12 extremely beneficial to the industry as a whole from both peak performance and reliability perspectives.

\section{Acknowledgements}

14 The authors acknowledge the financial supports by NASA-EPSCoR Program (Grant number

15 NNX10AN26A), National Science Foundation (NSF-EPSCoR Cooperative Agreement 1158862), and

16 Alabama-EPSCoR GRSP Program (Grant No. 130351).

\section{References}

18 1. Dransfield KA, Jain LK, Mai Y-W. On the effects of stitching in CFRPs - I. Mode I delamination

19 toughness. Compos Sci Technol 1998; 58: 815-827.

20 2. Velmurugan R, Solaimurugan S. Improvements in mode I interlaminar fracture toughness and in-plane

21 mechanical properties of stitched glass/polyester composites. Compos Sci Technol 2007; 67: 61-69.

22 3. Zhenf Y, Cheng X, Yasir B. Effect of Stitching on Plain and Open-hole Strength of CFRP Laminates.

23 Chinese Journal of Aeronautics 2012; 25: 473-484.

24 4. Lee SH, Kim H, Hang S, Cheoung S-K. Interlaminar fracture toughness of composite laminates with

25 CNT-enhanced nonwoven carbon tissue interleave. Compos Sci Technol 2012; 73: 1-8.

26 5. Arai M, Noro Y, Sugimoto K, Endo M. Mode I and mode II interlaminar fracture toughness of CFRP

27 laminates toughened by carbon nanofiber interlayer. Compos Sci Technol 2008; 68: 516-525. 
1 6. Garcia EJ, Wardle BL, Hart AJ. Joining prepreg composite interfaces with aligned carbon nanotubes.

2 Compos A 2008; 39: 1065-1070.

3 7. Yasaee M, Bond IP, Trask RS, Greenhalgh ES. Damage control using discrete thermoplastic film inserts.

$4 \quad$ Compos A 2012; 43: 978-989

5 8. Sadeghian R, Gangireddy S, Minaie B, Hsiao K-T. Manufacturing carbon nanofibers toughened

6 polyester/glass fiber composites using vacuum assisted resin transfer molding for enhancing the mode-I

7 delamination resistance. Compos A 2006; 37: 1787-1795.

8 9. Fan Zhihang, Santare MH, Advani SG. Interlaminar shear strength of glass fiber reinforced epoxy

9 composites enhanced with multi-walled carbon nanotubes. Compos A 2008; 39: 540-554.

10 10. Bekyarova E, Thostanson ET, Yu A, Kim H, Gao J, Tang J, Hahn HT, Chou T-W, Itkis ME, Haddon RC.

11 Multiscale carbon nanotube - carbon fiber reinforcement for advanced epoxy composites. Langmuir, $12 \quad 2007,23(7), 3970-3974$.

13 11. Wicks SS, Guzman de Villoria R, Wardle BL. Interlaminar and intralaminar reinforcement of composite 14 laminates with aligned carbon nanotubes. Compos Sci Technol 2010; 70: 20-28.

15 12. Wicks SS, Wang W, Williams MR, Wardle BL. Multi-scale interlaminar fracture mechanisms in woven 16 composite laminates reinforced with aligned carbon nanotubes. Compos Sci Technol 2014; 100: 128-135.

17 13. Fan $\mathrm{Z}$ and Advani SG. Characterization of orientation state of carbon nanotubes in shear flow, Polymer, $18 \quad 2005,46(14), 5232-5240$.

19 14. Hsiao K-T and Hickman GJS. Method For Manufacturing Nano Structurally Aligned Multi-Scale

20 Composites. US. Provisional Serial No. 61/958,485; PCT/2014/048406; WO/2015/017321 (2015).

21 15. "Pygrograf-III Carbon Nanofiber," e-Business Express, 2011. [Online]. Available:

$22 \mathrm{http}: / /$ pyrografproducts.com/nanofiber.html\#_PR-24-XT-PS_Data_Sheet.

23 16. ASTM D5528-01. Standard Test Method for Mode I Interlaminar Fracture Toughness of Unidirectional 24 Fiber-Reinforced Polymer Matrix Composites, ASTM International, West Conshohocken, PA, 2001. 


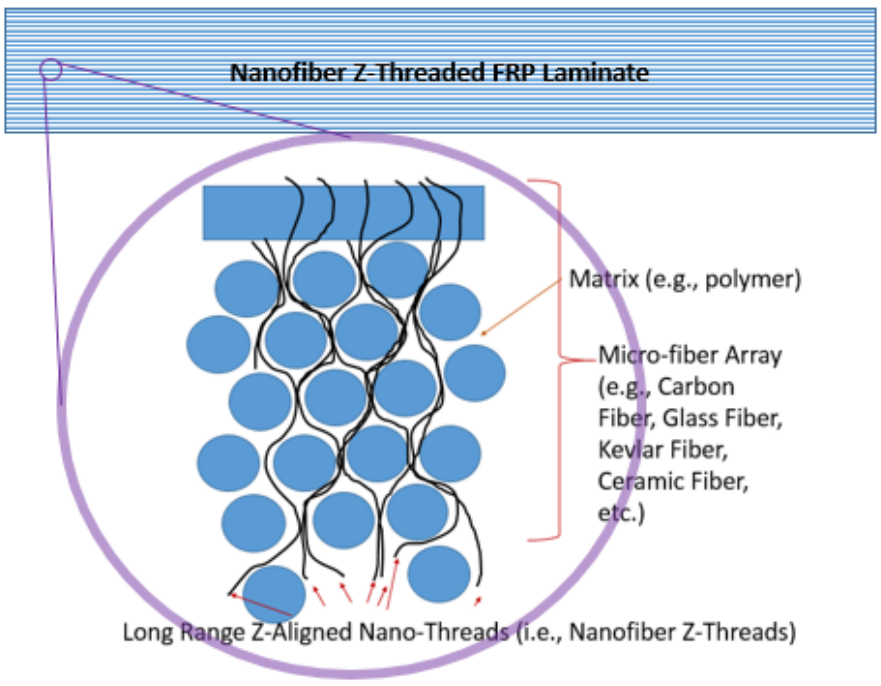

Fig. 1. Desired nanofiber $z$-threaded FRP presents the possibility of effective long-range reinforcement in the through-thickness direction (i.e., z-direction). Due to the long nanofiber length and zig-zag threading path, strong mechanical interlocks could be formed that yield highly effective z-direction reinforcement.

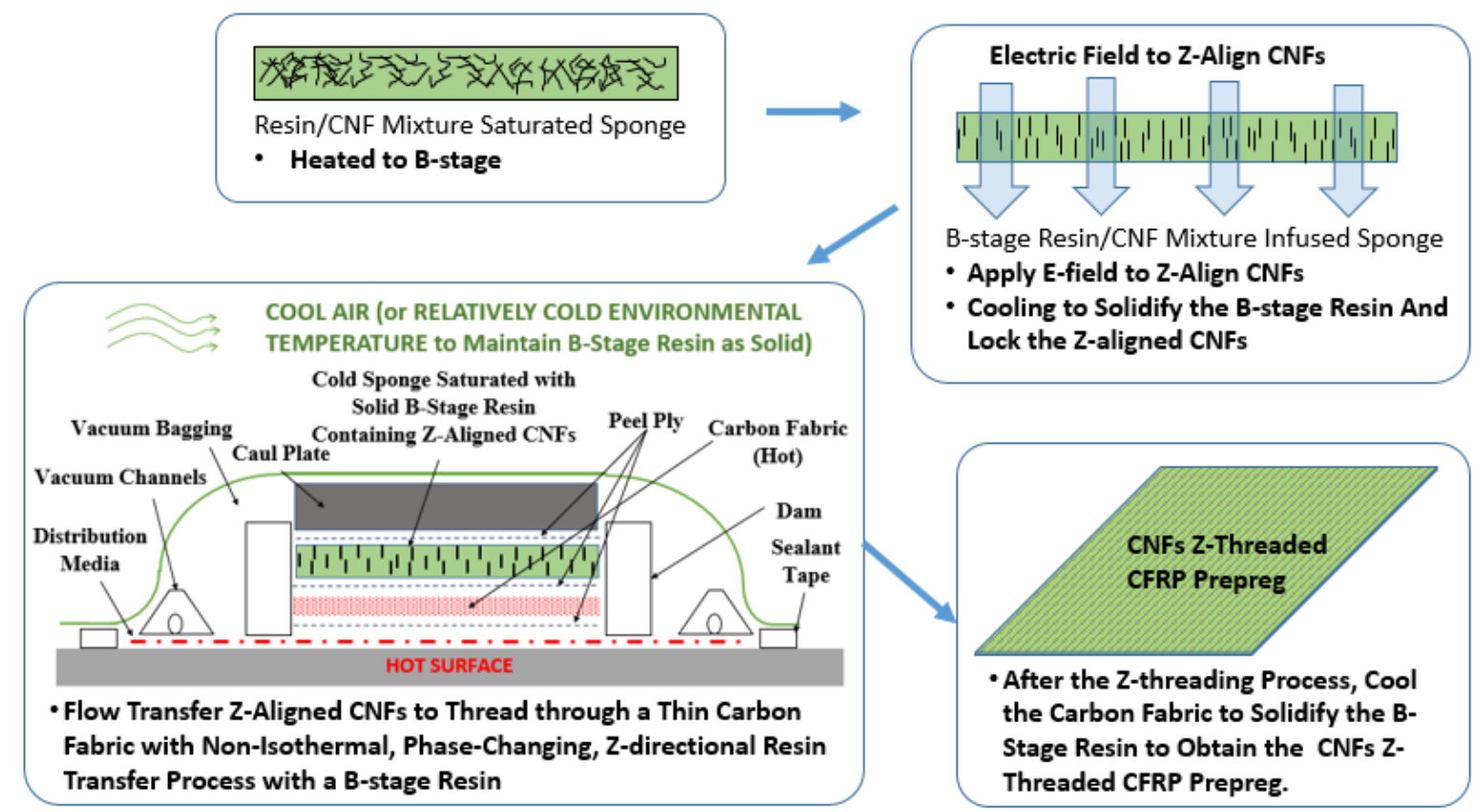

Fig. 2. Manufacturing of the CNF Z-threaded CFRP prepreg. Four majors steps are given as (1) saturate a thin sponge with B-stage CNF/resin mixture, (2) z-align CNFs in the sponge with electric field and solidify the Bstage resin to lock the CNF z-alignment, (3) apply non-isothermal, phase-changing, and z-directional resin transfer to thread the pre-z-aligned CNFs into a thin carbon fabric, (4) cool down the carbon fabric to solidify the B-stage resin and obtain CNFs z-threaded CFRP prepreg. 


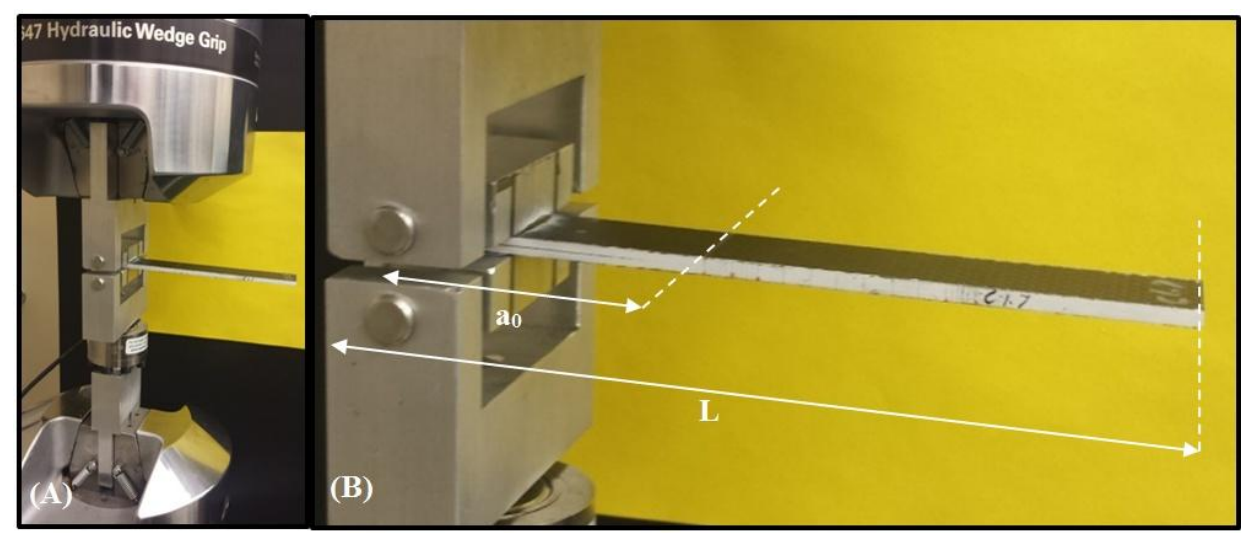

Fig. 3. Experimental test setup: (a) DCB fixture; (b) sample layout. Note that $a_{0}$ represents the initial crack length and $L$ depicts the overall sample length.

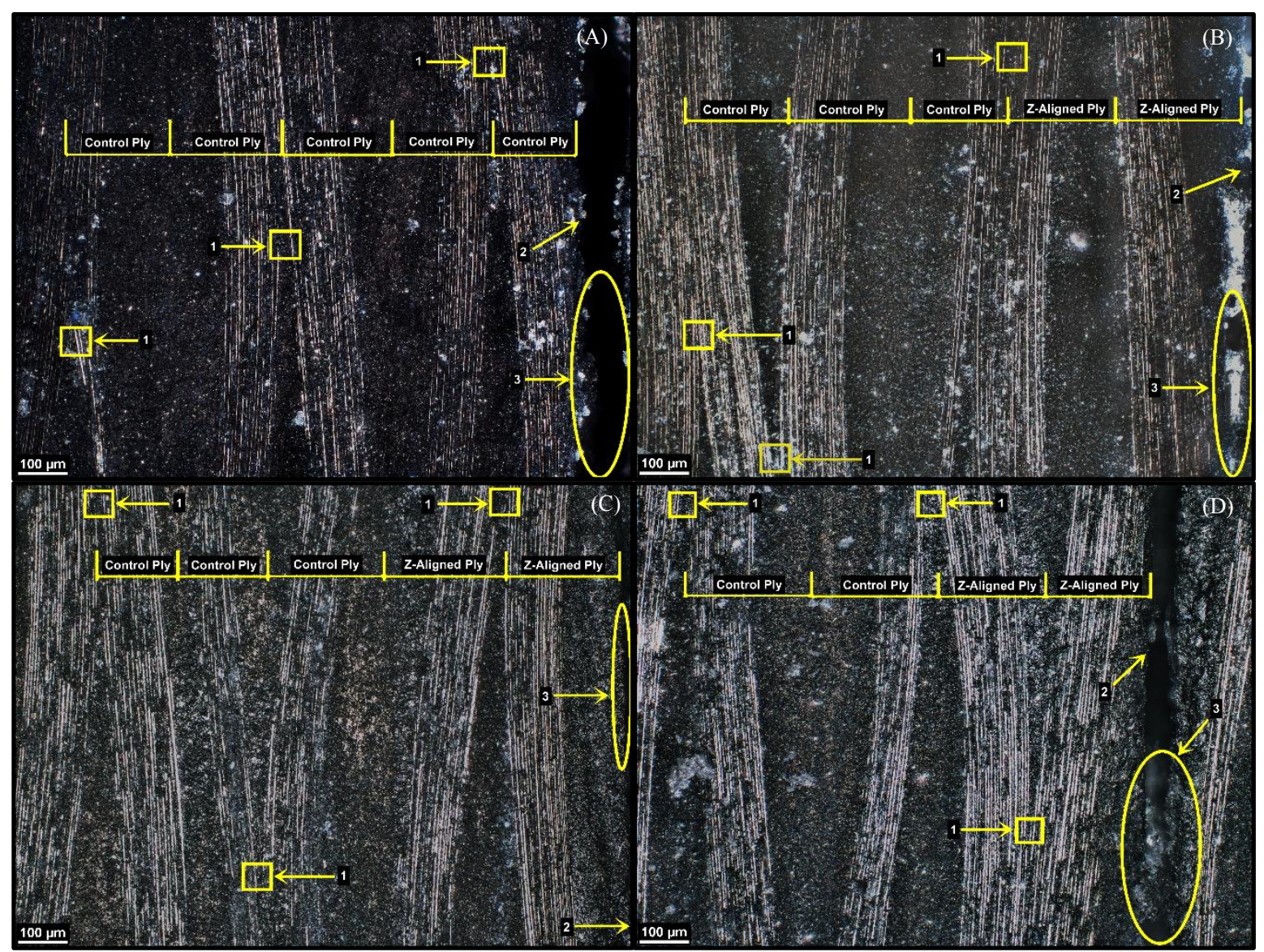

Fig. 4. Optical micrographs of the upper-half of symmetric CFRP laminate layer-by-layer structures taken at 100x magnification: (A) control CFRP; (B) 0.3wt\% UA-CFRP; (C) $0.3 \mathrm{wt} \%$ ZT-CFRP; (D) $0.6 \mathrm{wt} \% \mathrm{ZT}$ CFRP. In these micrographs, the provided annotations indicate: $1=$ interlaminar areas where the warp \& weft of successive plies within the laminate are joined; 2 = laminate mid-plane; $3=$ gap formed by removing PTFE pre-crack insert. If these CFRP laminates had incorporated interleaves, there would be visible resin-rich region separating the regions where warp and weft met (i.e., region-1). As shown, none of the samples contained any resin rich interleaving regions. 


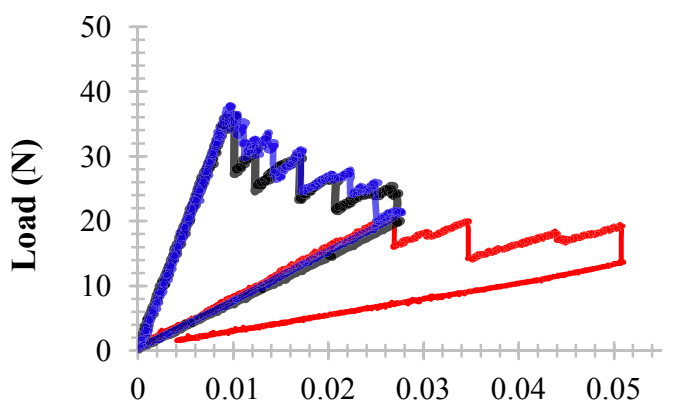

(A)

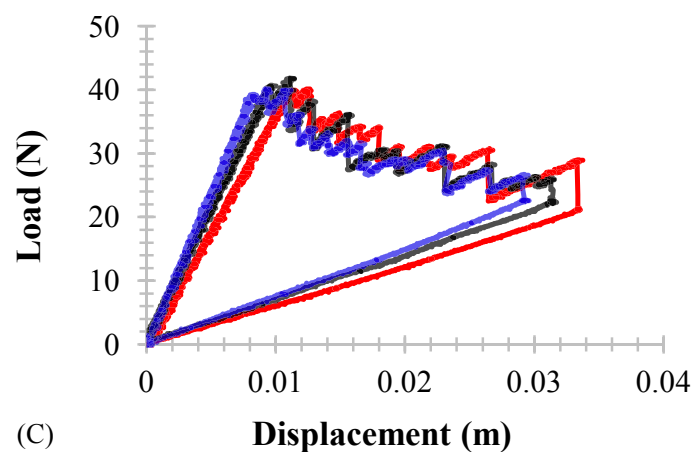

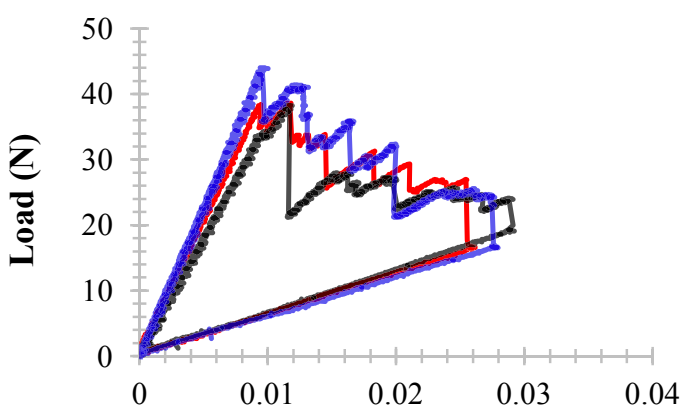

(B) Displacement $(\mathbf{m})$

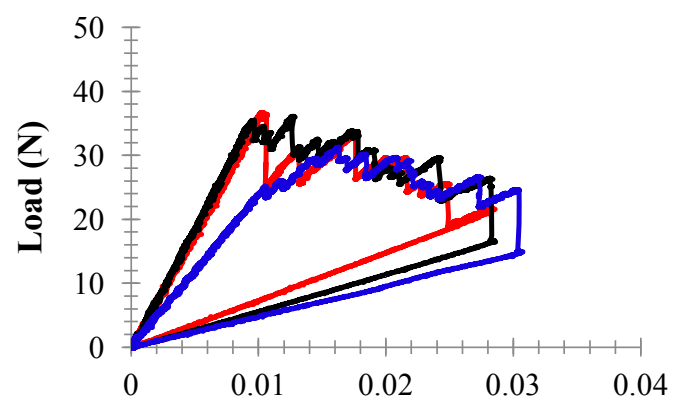

Displacement (m)

Fig. 5. DCB testing experimental data for: (A) control CFRPs; (B) $0.3 \mathrm{wt} \%$ UA-CFRPs; (C) $0.3 \mathrm{wt} \% \mathrm{ZT}$ CFRPs; (D) 0.6wt $\%$ ZT-CFRPs. Note that the data displayed here was captured during the reloading cycle.
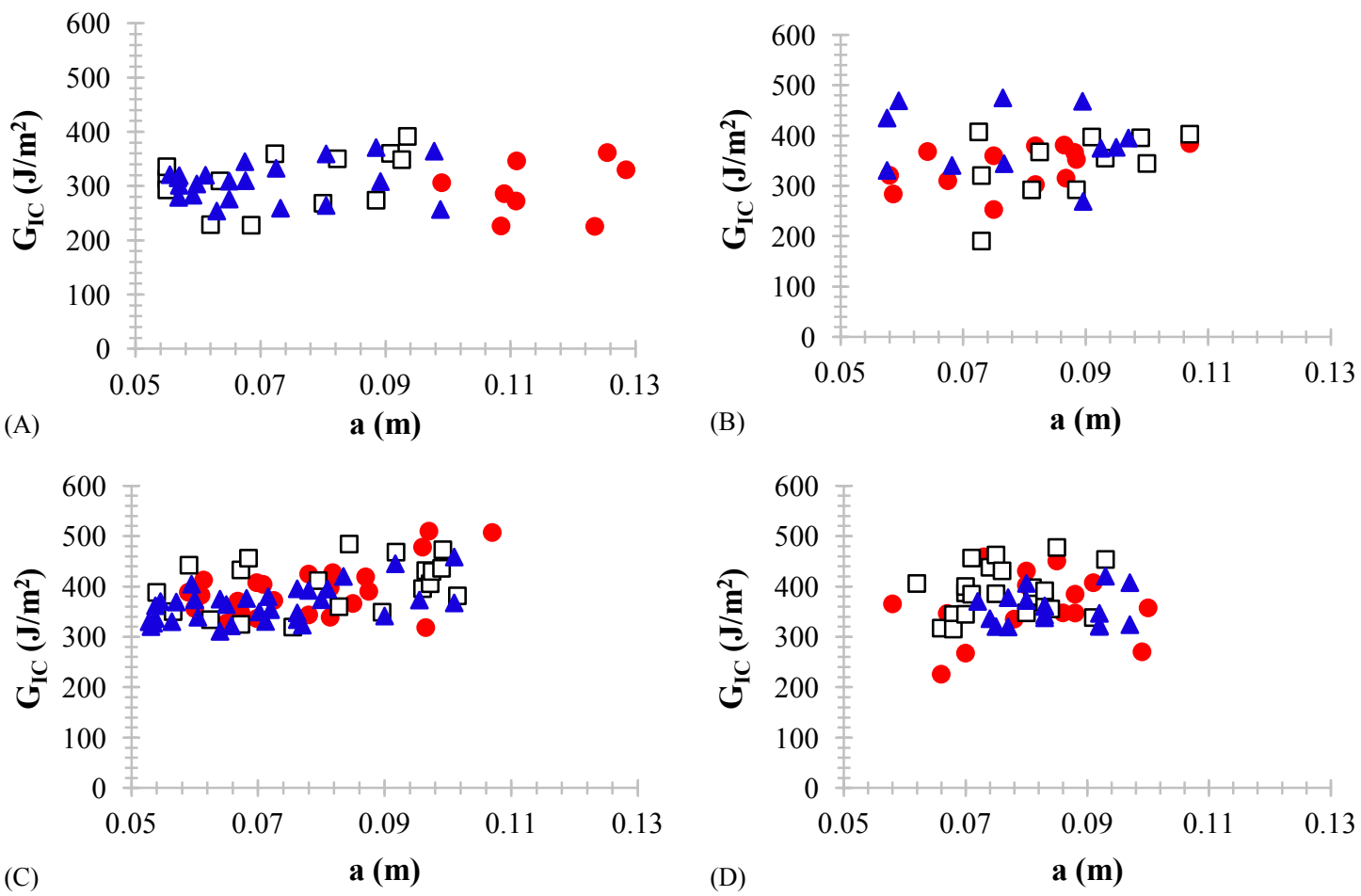

Fig. 6. DCB testing results using MCC approach for: (A) control CFRPs; (B) $0.3 w t \%$ UA-CFRPs; (C) $0.3 \mathrm{wt} \%$ ZT-CFRPs; (D) $0.6 \mathrm{wt} \%$ ZT-CFRPs. 


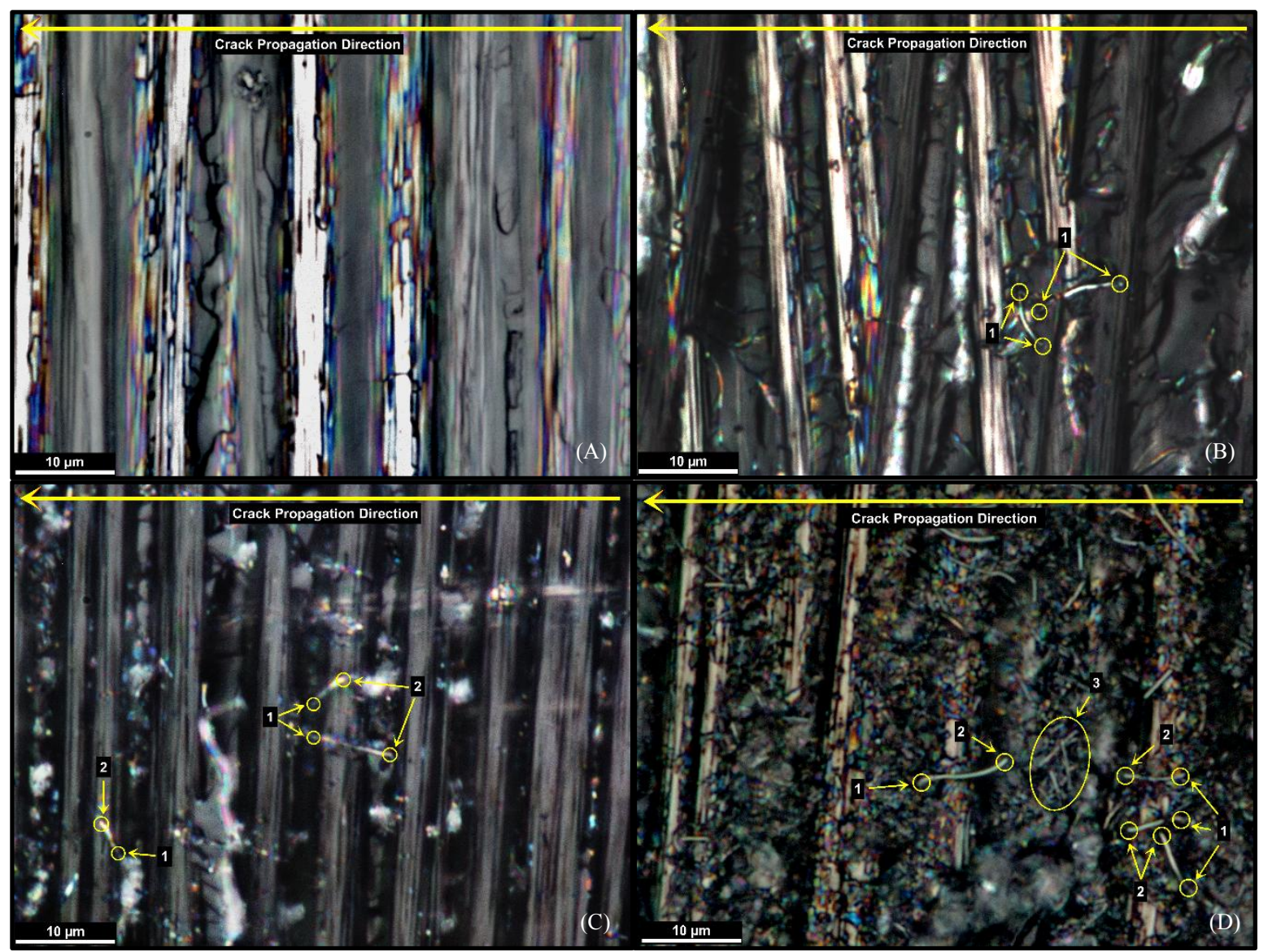

Fig. 7. Optical micrographs of the post-fracture delamination surface morphology taken at 2000x magnification: (A) control CFRP; (B) $0.3 \mathrm{wt} \%$ UA-CFRP; (C) $0.3 \mathrm{wt} \%$ ZT-CFRP; (D) 0.6wt \% ZT-CFRP. In these micrographs, the provided annotations indicate: $1=\mathrm{CNFs}$ rooted in matrix, $2=\mathrm{CNF}$ tips, $3=\mathrm{CNF}$ aggregation. A z-aligned or pseudo-z-aligned CNF should have a clear root in the matrix and a broken tip clearly exposed in air. In contrast to the one-root and one-tip formation, any CNF has either two roots or has a long-section submersed in the resin matrix parallel to the delamination surface is not z-aligned nor pseudo zaligned. 


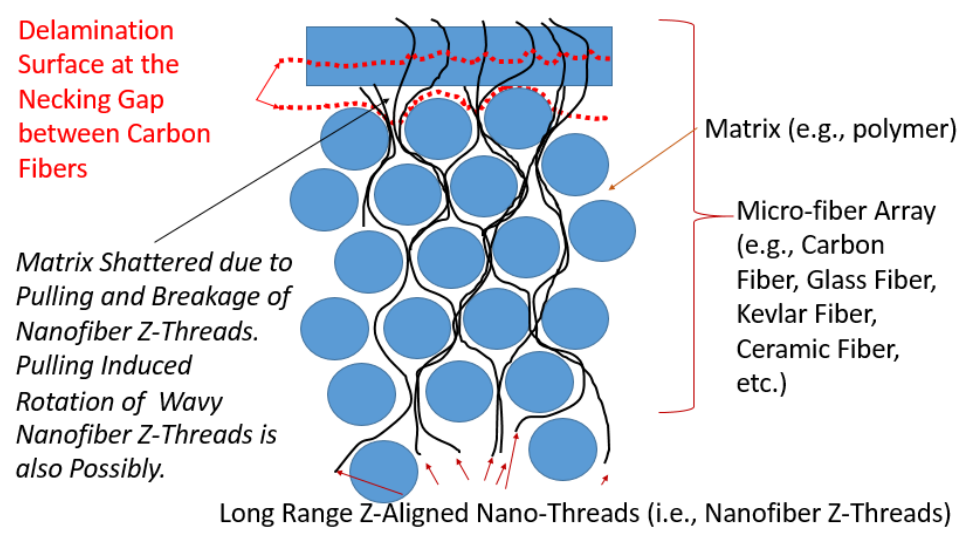

Fig. 8. Proposed CNFs z-threading patterns through a CFRP help the understanding of possible morphology of CNF z-threads (i.e., z-aligned CNFs) in a delaminated CFRP laminate. The delamination surface is likely to occur at the necking gap between carbon fibers. The matrix (i.e., resin) near the delamination surface is likely to be shattered due to the interaction with the CNF z-threads under tension. Also, according to the geometry, any broken CNF z-thread in the delamination surface can only have one root in the matrix and one broken tip. A CNF z-thread can possibly be broken near the root or broken with the shattered matrix.

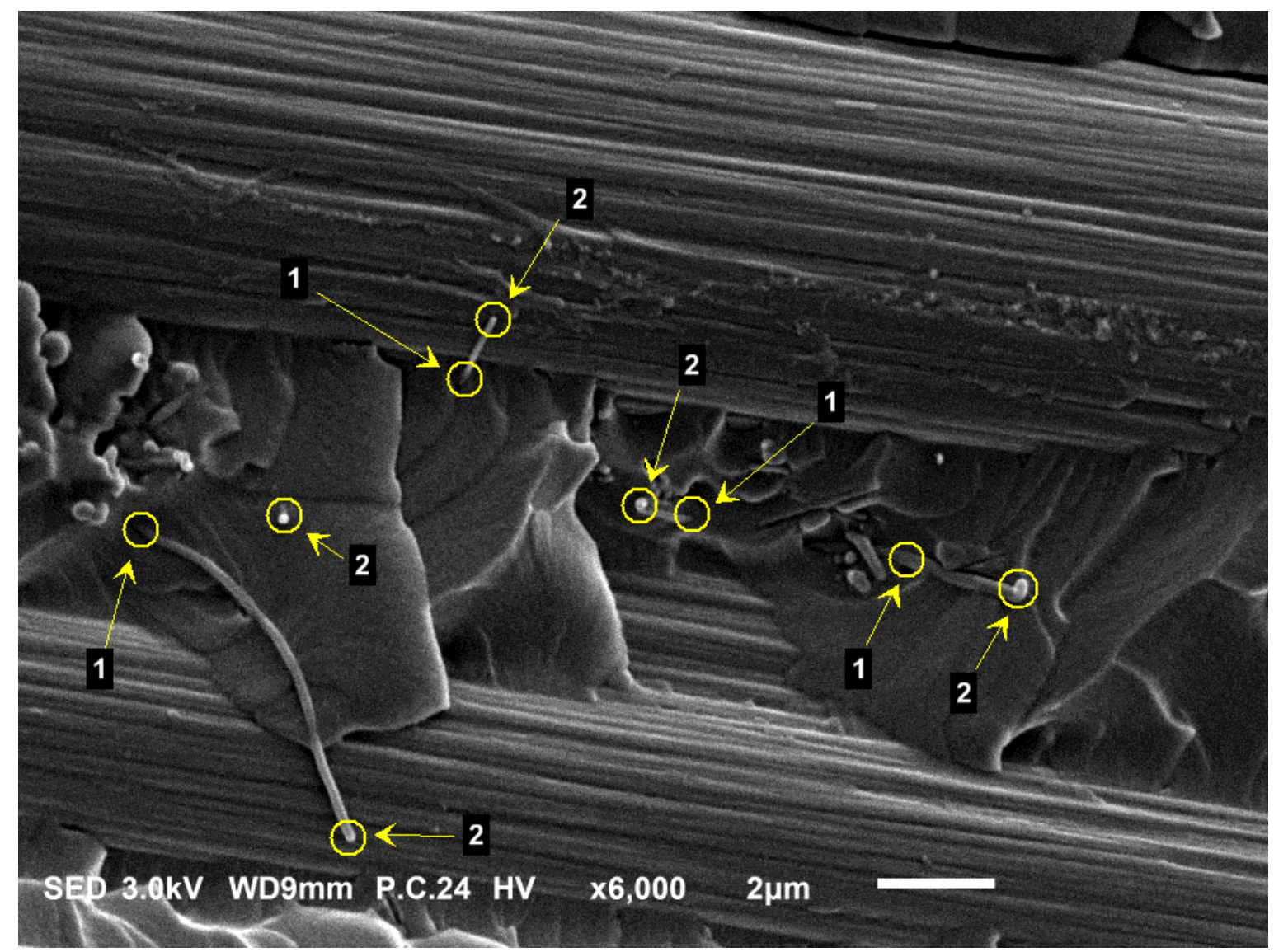

Fig. 9. SEM micrograph of $0.3 \mathrm{wt} \%$ ZT-CFRP post-fracture delamination surface showing intact aligned CNFs. In this micrograph, the provided annotations indicate: $1=\mathrm{CNFs}$ rooted in matrix, $2=\mathrm{CNF}$ tips. 


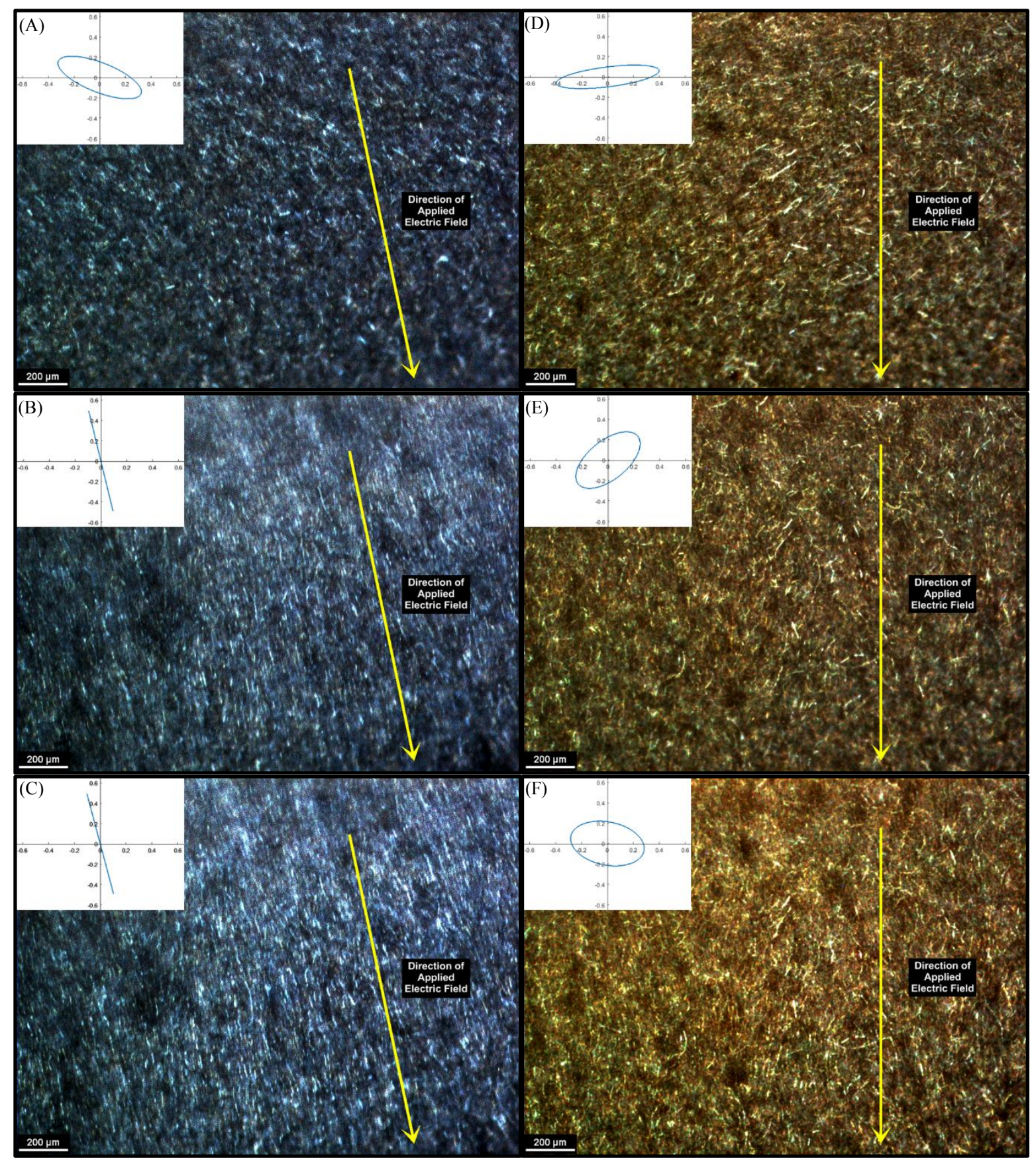

Fig. 10. Optical micrographs of $0.67 \mathrm{kV} / \mathrm{mm}$ electric field application $(6 \mathrm{~Hz}, 50 \%$ duty cycle) in uncatalyzed epoxy taken at 300x magnification of: (A) $0.3 \mathrm{wt} \%$ CNFs at 0 seconds, (B) 30 seconds, and (C) 60 seconds; (D) $0.6 \mathrm{wt} \% \mathrm{CNFs}$ at 0 seconds, (E) 30 seconds, and (F) 60 seconds. The $0.3 \mathrm{wt} \%$ sample was aligned by the electric field very effectively and one can also see some CNFs were straightened by the electric field from (B) to $(\mathrm{C})$, which can also be shown by the change in the shape and rotation of the ODF ellipses. On the other hand, the $0.6 \mathrm{wt} \%$ sample only achieved mediocre CNF alignment. (Note the videos for the two experiments can be found in " $0.3 \mathrm{wt} \%$ Alignment Video 5X Speed.mp4" and " $\underline{0.6 w t \%}$ Alignment Video 5X Speed.mp4" respectively) 
Table 1

OOA-VBO cure cycle used for all laminates.

\begin{tabular}{|ccc|}
\hline Time $(\min )$ & Temperature $\left({ }^{\circ} \mathrm{C}\right)$ & Vacuum \\
\hline 60 & $23($ Room Temp) & Yes \\
30 & 45 & Yes \\
120 & 120 & Yes \\
240 & 180 & No \\
\hline
\end{tabular}

\section{Table 2}

$\mathrm{G}_{\mathrm{IC}}$ sample statistics used to highlight performance variations between sample types.

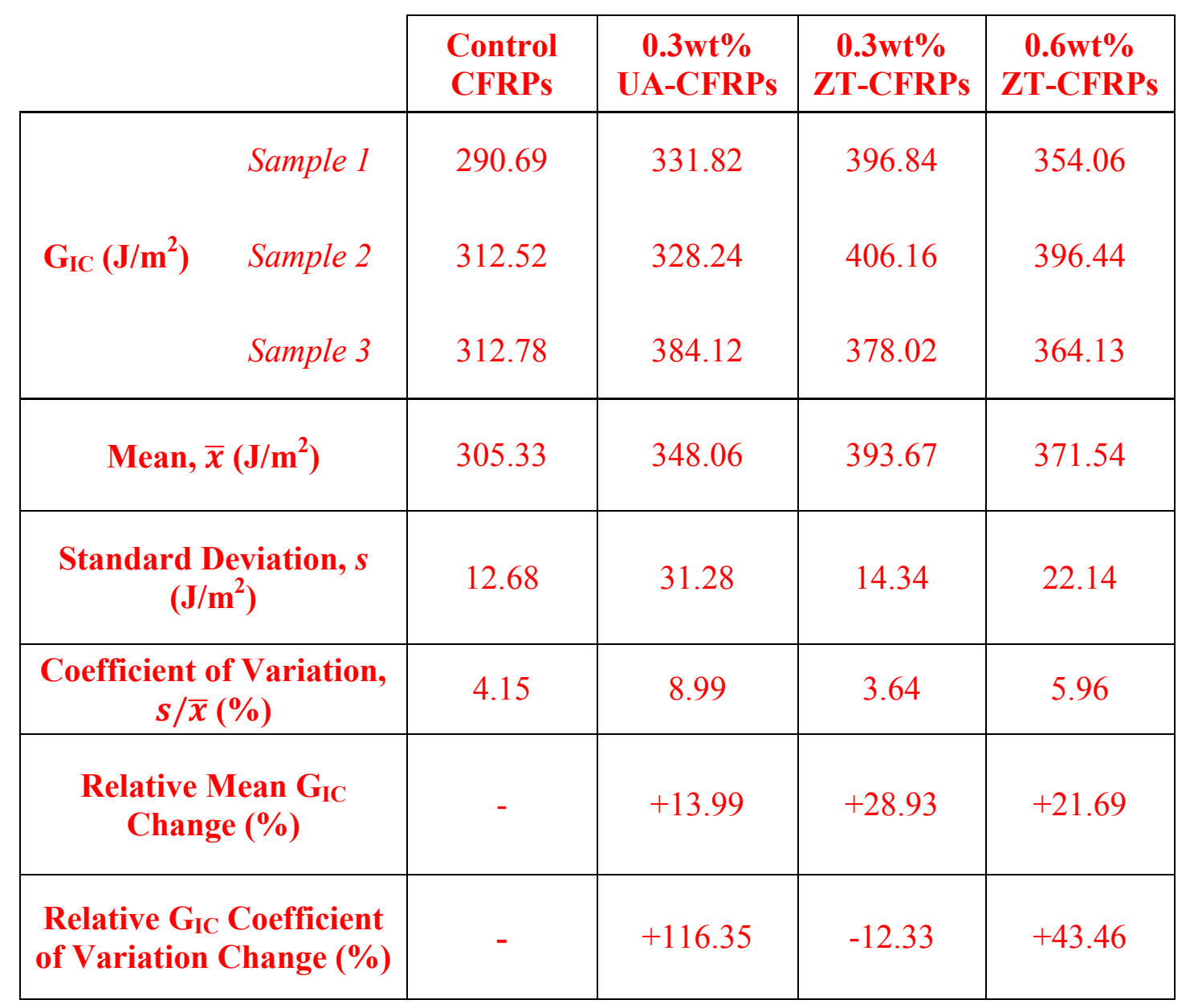




\section{List of Figures}

Fig. 1. Desired nanofiber z-threaded FRP presents the possibility of effective long-range reinforcement in the through-thickness direction (i.e., z-direction). Due to the long nanofiber length and zig-zag threading path, strong mechanical interlocks could be formed that yield highly effective z-direction reinforcement.

Fig. 2. Manufacturing of the CNF Z-threaded CFRP prepreg. Four majors steps are given as (1) saturate a thin sponge with B-stage CNF/resin mixture, (2) z-align CNFs in the sponge with electric field and solidify the B-stage resin to lock the CNF z-alignment, (3) apply non-isothermal, phase-changing, and z-directional resin transfer to thread the pre-z-aligned CNFs into a thin carbon fabric, (4) cool down the carbon fabric to solidify the B-stage resin and obtain CNFs z-threaded CFRP prepreg.

Fig. 3. Fig. 3. Experimental test setup: (a) DCB fixture; (b) sample layout. Note that a0 represents the initial crack length and $\mathrm{L}$ depicts the overall sample length.

Fig. 4. Optical micrographs of the upper-half of symmetric CFRP laminate layer-by-layer structures taken at 100x magnification: (A) control CFRP; (B) 0.3wt\% UA-CFRP; (C) 0.3wt \% ZT-CFRP; (D) 0.6wt\% ZT-CFRP. In these micrographs, the provided annotations indicate: $1=$ interlaminar areas where the warp \& weft of successive plies within the laminate are joined; 2 = laminate mid-plane; $3=$ gap formed by removing PTFE pre-crack insert. If these CFRP laminates had incorporated interleaves, there would be visible resin-rich region separating the regions where warp and weft met (i.e., region1). As shown, none of the samples contained any resin rich interleaving regions.

Fig. 5. DCB testing experimental data for: (A) control CFRPs; (B) $0.3 w t \%$ UA-CFRPs; (C) $0.3 w t \%$ ZTCFRPs; (D) 0.6wt\% ZT-CFRPs. Note that the data displayed here was captured during the reloading cycle.

Fig. 6. DCB testing results using MCCMBT approach for: (A) control CFRPs; (B) 0.3wt\% UA-CFRPs; (C) $0.3 \mathrm{wt} \%$ ZT-CFRPs; (D) $0.6 \mathrm{wt} \%$ ZT-CFRPs. 
Fig. 7. Optical micrographs of the post-fracture delamination surface morphology taken at 2000x magnification: (A) control CFRP; (B) 0.3wt\% UA-CFRP; (C) 0.3wt\% ZT-CFRP; (D) 0.6wt\% ZTCFRP. In these micrographs, the provided annotations indicate: $1=\mathrm{CNFs}$ rooted in matrix, $2=\mathrm{CNF}$ tips, $3=\mathrm{CNF}$ aggregation. A z-aligned or pseudo-z-aligned CNF should have a clear root in the matrix and a broken tip clearly exposed in air. In contrast to the one-root and one-tip formation, any CNF has either two roots or has a long-section submersed in the resin matrix parallel to the delamination surface is not $\mathrm{z}$-aligned nor pseudo $\mathrm{z}$-aligned.

Fig. 8. Proposed CNFs z-threading patterns through a CFRP help the understanding of possible morphology of CNF z-threads (i.e., z-aligned CNFs) in a delaminated CFRP laminate. The delamination surface is likely to occur at the necking gap between carbon fibers. The matrix (i.e., resin) near the delamination surface is likely to be shattered due to the interaction with the CNF z-threads under tension. Also, according to the geometry, any broken CNF z-thread in the delamination surface can only have one root in the matrix and one broken tip. A CNF z-thread can possibly be broken near the root or broken with the shattered matrix.

Fig. 9. SEM micrograph of $0.3 \mathrm{wt} \%$ ZT-CFRP post-fracture delamination surface showing intact aligned CNFs. In this micrograph, the provided annotations indicate: $1=\mathrm{CNFs}$ rooted in matrix, $2=\mathrm{CNF}$ tips.

Fig. 10. Optical micrographs of $0.67 \mathrm{kV} / \mathrm{mm}$ electric field application $(6 \mathrm{~Hz}, 50 \%$ duty cycle) in uncatalyzed epoxy taken at 300x magnification of: (A) $0.3 \mathrm{wt} \%$ CNFs at 0 seconds, (B) 30 seconds, and (C) 60 seconds; (D) $0.6 \mathrm{wt} \%$ CNFs at 0 seconds, (E) 30 seconds, and (F) 60 seconds. The same group of CNFs are highlighted and tracked with yellow squares for easy comparison. The $0.3 \mathrm{wt} \%$ samples was aligned by the electric field very effectively and one can also see some CNFs were straightened by the electric field from (B) to (C), which can also be shown by the change in the shape and rotation of the ODF ellipses. On the other hand,- tThe $0.6 \mathrm{wt} \%$ sample only has mediocre CNF alignment results by the electric field. (Note the videos for the two experiments can be found in " $0.3 \mathbf{w t} \%$ $\underline{\text { Alignment Video 5X Speed.mp4" and "0.6wt\% Alignment Video 5X Speed.mp4" respectively) }}$ 
Table caption

\section{List of Tables}

Table 1. OOA-VBO cure cycle used for all laminates.

Table 2. GIC sample statistics used to highlight performance variations between sample types.

12
13
14
15
16
17
18
19
20
21
22
23
24
25
26
27
28
29
30
31
32
33
34
35
36

